\title{
Protocol \\ Study Protocol on the Validation of the Quality of Sleep Data from Xiaomi Domestic Wristbands
}

\author{
Patricia Concheiro-Moscoso ${ }^{1,2}{ }^{\mathbb{D}}$, Francisco José Martínez-Martínez ${ }^{1}{ }^{(D)}$, María del Carmen Miranda-Duro ${ }^{1,2}$ (D), \\ Thais Pousada ${ }^{1,2} \mathbb{D}$, Laura Nieto-Riveiro ${ }^{1,2} \mathbb{D}$, Betania Groba ${ }^{1,2} * \mathbb{D}$, Francisco Javier Mejuto-Muiño ${ }^{3}$ and \\ Javier Pereira ${ }^{1,2}$ (D)
}

\section{check for}

updates

Citation: Concheiro-Moscoso, P.; Martínez-Martínez, F.J.;

Miranda-Duro, M.d.C.; Pousada, T.;

Nieto-Riveiro, L.; Groba, B.;

Mejuto-Muiño, F.J.; Pereira, J. Study

Protocol on the Validation of the Quality of Sleep Data from Xiaomi Domestic Wristbands. Int. J. Environ. Res. Public Health 2021, 18, 1106. https://doi.org/10.3390/ ijerph18031106

Academic Editor: Jennifer L. Scheid Received: 26 November 2020

Accepted: 23 January 2021

Published: 27 January 2021

Publisher's Note: MDPI stays neutral with regard to jurisdictional claims in published maps and institutional affiliations.

Copyright: (c) 2021 by the authors. Licensee MDPI, Basel, Switzerland. This article is an open access article distributed under the terms and conditions of the Creative Commons Attribution (CC BY) license (https:// creativecommons.org/licenses/by/ $4.0 /)$.
1 CITIC, TALIONIS Group, Elviña Campus, Universidade da Coruña (University of A Coruña), 15071 A Coruña, Spain; patricia.concheiro@udc.es (P.C.-M.); f.martinezm@udc.es (F.J.M.-M.); carmen.miranda@udc.es (M.d.C.M.-D.); thais.pousada.garcia@udc.es (T.P.); laura.nieto@udc.es (L.N.-R.); javier.pereira@udc.es (J.P.)

2 Faculty of Health Sciences, Oza Campus, Universidade da Coruña (University of A Coruña), 15071 A Coruña, Spain

3 Clinical Neurophysiology Service, Hospital San Rafael, 15009 A Coruña, Spain; fmejmui@gmail.com

* Correspondence: b.groba@udc.es; Tel.: +34-8-8101-5870

\begin{abstract}
Background: Sleep disorders are a common problem for public health since they are considered potential triggers and predictors of some mental and physical diseases. Evaluating the sleep quality of a person may be a first step to prevent further health issues that diminish their independence and quality of life. Polysomnography (PSG) is the "gold standard" for sleep studies, but this technique presents some drawbacks. Thus, this study intends to assess the capability of the new Xiaomi Mi Smart Band 5 to be used as a tool for sleep self-assessment. (2) Methods: This study will be an observational and prospective study set at the sleep unit of a hospital in A Coruña, Spain. Forty-three participants who meet the inclusion criteria will be asked to participate. Specific statistical methods will be used to analyze the data collected using the Xiaomi Mi Smart Band 5 and PSG. (3) Discussion: This study offers a promising approach to assess whether the Xiaomi Mi Smart Band 5 correctly records our sleep. Even though these devices are not expected to replace PSG, they may be used as an initial evaluation tool for users to manage their own sleep quality and, if necessary, consult a health professional. Further, the device may help users make simple changes to their habits to improve other health issues as well. Trial registration: NCT04568408 (Registered 23 September 2020).
\end{abstract}

Keywords: sleep; health promotion; daily life activities; occupation; polysomnography; Xiaomi Mi Smart Band 5; wearable technology; participatory health; internet of things

\section{Introduction}

Sleep is an occupational area that has considerable implications on our daily life [1,2]. Thus, sleep disorders have become one of the most important common problems in public health [3]. The prevalence of sleep disorders increases with age [4], even though they can appear at any life stage. Bad quality and quantity of sleep, sleep arousals, and a strong will to take diurnal naps are the main complaints among the general population $[5,6]$. Epidemiological studies indicate that between $20 \%$ and $48 \%$ of adults between 34 and 60 years old have difficulties initiating and maintaining sleep [7]. In fact, insomnia, which is the most common sleep disorder, is present in $30-45 \%$ of the population [8].

Several studies state that sleep disorders are related to health status and quality of life in the general population [9], leading to difficulties in the performance of daily life activities, which in turn causes physical exhaustion, low productivity, a greater risk of falling, mood problems, and diurnal sleep, among many others [10-14].

These factors related to sleep disorders cause a direct and indirect economic burden, causing public health costs to rise $[15,16]$. Different studies have shown a direct relation 
between sleep disorders and costs in primary care and hospitals. Lee et al. indicated that the population suffering from sleep disorders regularly attends the emergency room to consult professional health workers, or demands telematic assistance [9]. This is due to subjective complaints of sleep, along with factors related to stress, working pressure, and other chronic diseases $[17,18]$.

Diverse sleep disorders are diagnosed using different tests that assess the quality and quantity of sleep $[19,20]$. Objective and valid diagnostic tests that evaluate the quality and quantity of sleep are a previous requisite for the control of sleep disorders [21]. Polysomnography (PSG) is considered by the scientific community as the most reliable test for the measurement of sleep parameters [22,23]. However, it has some clear drawbacks, such as its costs, operation, invasiveness, and time needed for its use [24,25]. To address these problems, actigraphy technology was designed. Actigraphy consists of the measure of sleep parameters using a wearable device created for clinical use [26]. Several studies have found similar measures from both actigraphy and PSG, proving its fitness for these studies [27-29]. Although actigraphy has become a clinically useful tool to test sleep disorders, it cannot provide feedback to people because it purpose is mainly of a research nature [26]. Thus, inspired by the actigraphy device, researchers have recently focused on the validation of different tools for sleep self-assessment, such as activity wristbands [30].

In recent years, activity wristbands have become widely accepted among the population due to their low price, easy use, smart design, and feedback that they offer to users through their fast sync with smartphones, boosting participatory health [31-33]. These devices have already reported benefits in clinics, as they reduce care burdens and facilitate the early diagnosis of health disorders [34]. They also allow the general population to acquire information on their sleep quality and physical activity, which may help in the early detection or prevention of symptoms related to sleep alterations [35-37].

Despite being limited, some studies highlight the importance of the validation of these devices not only from a technological point of view, but also for health promotion, since, by allowing sleep self-assessment, they are tightly related to improvements in life quality and daily functioning [38-41].

Recent studies have compared the measurements of these wristbands with those of actigraphy and PSG techniques. PSG involves different types of electrodes (electroencephalogram (EEG), electrooculogram (EOG), electromyogram (EMG), and electrocardiogram (ECG)) to classify the stages of sleep [42]. A few studies have shown that the usability of an ECG signal can determine the stages of sleep [43-46]. Nevertheless, some have considered the need for further research due to the insufficient quality of this classification compared to the combination of the other electrodes $[47,48]$.

In general, wearable devices can determine the stages of sleep through a heart rate (HR) sensor, which measures the HR, and an accelerometer that detects movement. Previously validated wearable devices have shown a high precision and sensitivity, but also a low specificity and poor agreement in sleep-stage classification when compared with PSG $[20,32,33,49,50]$. It would be logical to expect these devices to measure sleep more accurately; however, according to these studies, there is a wide range of improvements that can still be accomplished [51,52]. As a result, this protocol has been designed to validate the Xiaomi Mi Smart Band 5 due to its low cost and high acceptance among users, as it is the best-selling fitness tracker [53]. This device has the capability of tracking the activity and HR of users, and classifies the user's state as being awake or in light sleep, deep sleep, or REM sleep [54].

Therefore, this research focuses on determining whether sleep stages recorded by the new Xiaomi Mi Band 5 can effectively replace PSG sleep-stage classification in sleep-study participants. To this end, the following features will be calculated for both devices: total sleep time, sleep efficiency, sleep latency, and wake after sleep onset. Sleep stages will also be recorded. 
Thus, the primary purpose of the present study is to validate the quality of the data generated by Xiaomi wearables when compared with the PSG technique from a hospital sleep unit.

The secondary objectives are: (1) to determine the total sleep time, sleep efficiency, sleep latency, and wake after sleep onset from both the wearable device and PSG; and (2) to examine whether the classification of sleep stages provided by wearable devices is comparable with classifications based on PSG.

\section{Materials and Methods}

\subsection{Study Design}

This is a pilot study that aims "to demonstrate that the expected measures, data collecting instruments, and their management system are viable and effective" [55]. This is an observational and prospective study, which means that different variables from the population of interest will be observed and recorded without any direct intervention, in order to establish causality associations between these variables. It will be considered a longitudinal study, with a timeframe of six months, in which participants will stay one night at the sleep unit. They will be monitored to record their sleep through PSG and the Xiaomi wristband.

This study protocol follows the SPIRIT 2013 checklist for study protocols for clinical trials (See File S1) [56].

\subsection{Study Settings}

The setting of this study will be the sleep unit of a hospital in A Coruña, Spain. This service includes a special consulting room where different sleep disorders are diagnosed and treated. Some of these disorders are sleep apnea, insomnia, restless-legs syndrome, narcolepsy, the delay and advance of sleep stages, sleep terrors, somnambulism, and bruxism. The sleep laboratory is equipped to perform different diagnostic tests: nocturnal and diurnal PSG, multiple latency tests, immobilization tests, and wakefulness maintenance tests.

\subsection{Eligibility Criteria}

Sample selection will be performed through an intentional sampling based on inclusion and exclusion criteria.

Participant inclusion criteria: (a) having attended the sleep unit to have any diagnostic test done; (b) having declared an age that is equal to or higher than 18 years old.

Participant exclusion criteria: (a) having a significant health complication that hinders active participation in the study; (b) suffering from skin hypersensitivity or an allergic reaction due to the materials of the wristbands that will be used in the study.

\subsection{Recruitment Process}

This project will be conducted in the sleep unit of a hospital that, regardless of this study, people attend to have PSG tests done with the aim of detecting possible alterations in their sleep. The participants will be assisted by, at a minimum, the clinical neurophysiologist responsible for the sleep unit and a member of the research team. In the first meeting with each of the users, the clinical neurophysiologist responsible for recruiting the participants and monitoring them will inform them, if they meet the selection criteria, about the possibility of participating in the study and the implications of their participation, i.e., the use of an activity bracelet during the day or night and what is involved in the PSG test that will be performed on them. In addition, the mechanisms that will be followed to guarantee their anonymity and the confidentiality of their data will be clearly stated to the participants.

After the main characteristics of the research have been explained, each potential participant will be given an information sheet so that they can consult the information and make a decision before the test is carried out. Once the users return to the unit for 
testing, any doubts or queries will be resolved by the clinical neurophysiologist, and the informed consent document will be signed, if applicable, by the responsible professional and the person who expresses their final interest in participating in the study. In addition, if the participant has reading and writing difficulties, a witness must be present during the entire procedure to confirm that all the ethical processes have been respected by the research team. On the day of the PSG test, participants will arrive three hours before the test to become acquainted with the room, and will turn the lights on and off based on their sleep pattern. The specialized technical staff will control the test and will attend to any possible patient demands. Figure 1 shows the recruitment and the assessment process.

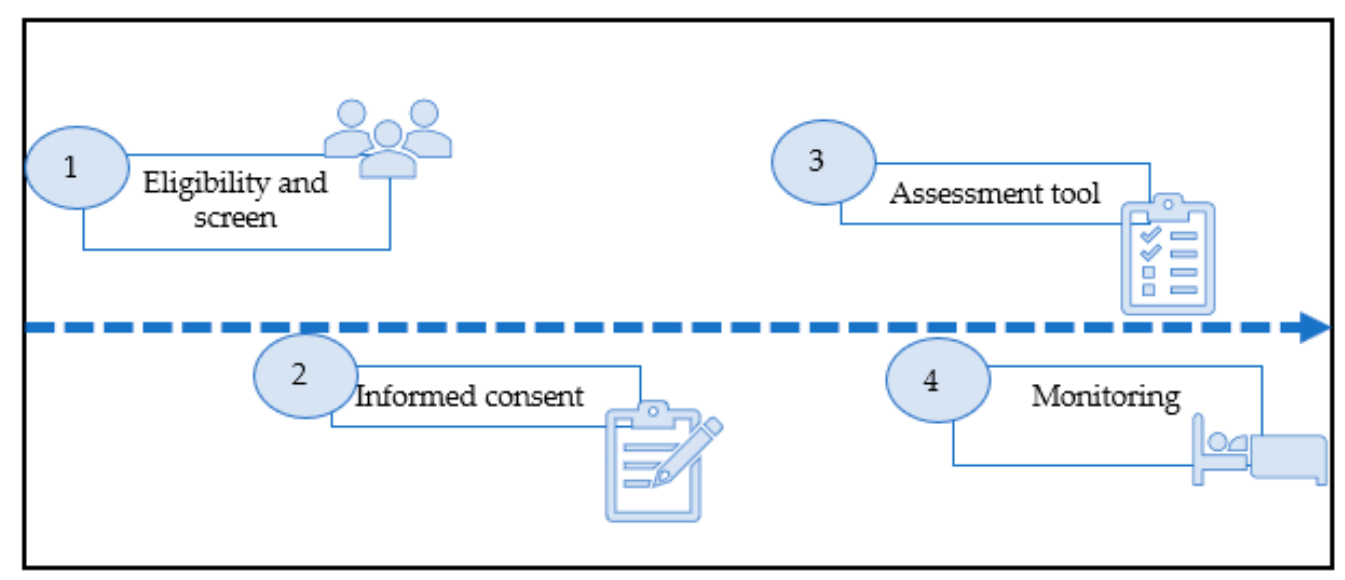

Figure 1. The recruitment and assessment process.

\subsection{Justification of Sample Size}

Differences equal or greater than $15 \mathrm{~min}$ in deep-sleep time between the measures of both methods are considered clinically relevant. Accepting a 0.05 alpha risk and a 0.1 beta risk ( $90 \%$ power) in a bilateral contrast, 43 subjects are needed to detect a difference that is equal to or greater than $15 \mathrm{~min}$. Conservatively, a standard deviation of 30 for mean differences is assumed, according to previous studies [21,50,57].

\subsection{Outcomes}

The outcomes will be the sleep-stage identification of PSG and the Xiaomi Mi Smart Band 5, and measurements of total sleep time, sleep efficiency, sleep latency, and wake after sleep onset.

\subsection{Data Collection and Management}

The study will focus on examining the following variables (Table 1): time in bed, hours of light, deep and Rapid-Eye-Movement (REM) sleep, sleep efficiency, sleep latency, wake after sleep onset, and HR. Additionally, the Pittsburgh Sleep Quality Index (PSQI) scale and a sociodemographic questionnaire will be used to assess participants' self-awareness of their sleep.

Table 1. Summary of the features of interest for our study.

\begin{tabular}{ccc}
\hline Variable & Description & Dimension \\
\hline Time in bed (TIB) & Total time the patient is laying down & $\min$ \\
Sleep onset latency (SOL) & Length of time from full wakefulness to sleep & $\min$ \\
Wake after sleep onset (WASO) & Periods of wakefulness after defined sleep onset & $\min$ \\
Sleep efficiency (SE) & Time asleep/TIB $\times 100$ & $\%$ \\
Light sleep & N1 + N2 sleep stages & min \\
Deep sleep & N3 sleep stages & min \\
Rapid Eye Movement (REM) & - & min \\
sleep & & \\
\hline
\end{tabular}


The sociodemographic questionnaire will collect different data: gender, year of birth, weight, height, handedness, sleep pathologies, and the assessment test. These data will be pseudonymized and transferred by the hospital to the research group with the prior consent of the participant.

The PSQI will evaluate subjective sleep quality. This questionnaire consists of 24 items about the area of sleep. This tool analyzes the quantity, quality, duration, latency, and efficiency of sleep [58].

The objective sleep data will be obtained through two different devices:

Xiaomi Mi Smart Band 5: This is a wearable device focused on health and sport that measures biomedical parameters of users, among which HR and sleep measurements are the most remarkable. The wristband also requires certain personal data from the user to precisely calculate the activity, such as age, height, weight, gender, handedness, and wristband location. Wristband data will be obtained from the Mi Fit application, which is the native app of the Xiaomi Mi Band wristband. It is necessary to connect the wristband via Bluetooth to a smartphone of general use for the group, creating generic emails for each of the wristbands. The features of interest will be calculated from the data shown in this app [32].

PSG: Data obtained from diurnal and nocturnal PSG will be collected, as this is the standard test for patients who attend to the hospital's sleep unit. PSG measures sleep cycles and stages (N1, N2, N3, and REM sleep) by recording different variables, such as brain waves, eye movement, skeletal muscle activity, heart frequency and rhythm, blood pressure, oxygen level in the blood, breathing patterns, body position, limb movements, and whether there is snoring or other noise [59]. PSG parameters will be scored in $30 \mathrm{~s}$ epochs according to the American Academy of Sleep Medicine (AASM) guidelines [60], and PSG recordings will be exported in the European Data Format+ format [61] and interpreted by a doctor specialized in the study of sleep. Thus, sleep-stage classification based on PSG will be considered the "gold standard" for this study.

The data will be pseudonymized at the moment they are recorded; thus, the confidentiality of the data collected and the anonymity of each participant will be maintained. Once the project has ended, each participant's data will be stored for future studies if they provide consent.

\subsection{Data Analysis}

The analysis will be performed by using the statistical software R. Numeric variables will be expressed as mean (M) and standard deviation (SD), including the range, minima, and maxima. Beyond simple data and study variable descriptions, inferential analysis will be performed in order to determine possible significant relationships between the variables of the study.

A paired-sample Student's t-test will be used to compare the means of the different sleep parameters of interest. T-test effect sizes are 0.2 (small effect), 0.5 (moderate effect), and 0.8 (large effect), so if the means of two groups do not differ by a 0.2 standard deviation, the difference is trivial, even if there is a statistically significant relationship. [62]. BlandAltman plots will be used to assess the concordance between both devices for each of the sleep parameters (total sleep time, sleep efficiency, and wake after sleep onset). A positive bias indicates that the device tends to underestimate a variable when compared with PSG. A negative bias indicates that a sleep variable is overestimated [63]. Point estimations will be calculated, as well as their $95 \%$ confidence interval.

An epoch-by-epoch (EBE) ( $\mathrm{min} \times \mathrm{min})$ analysis will be performed in order to calculate the sensitivity (the proportion of epoch segments identified as a sleep state by the PSG that are correctly identified by Xiaomi), specificity (the proportion of epoch segments identified as a waking state by the PSG that are correctly identified by the Xiaomi wristband), and the level of agreement between both devices for light sleep identification (the proportion of PSG-classified N1 + N2 stages identified as light sleep by the Xiaomi wristband), deep sleep identification (the proportion of PSG N3 + N4 stages identified as deep sleep by 
the Xiaomi wristband), and REM sleep identification (proportion of PSG-classified REM stages identified as REM sleep by the Xiaomi wristband) [64]. Data analysis will include the cleaning or preprocessing, description, and processing of the stored data. The final aim of this workflow will be to obtain useful information that can be used for decision-making. During preprocessing, the wrong values of the dataset will be removed or corrected to avoid bias in the results. Subsequently, obtaining a descriptive statistic study that summarizes relations and distributions in a simple way will help us to know which processing strategy needs to be taken. When processing, information extraction will be performed.

\subsection{Ethics and Dissemination}

This study protocol was approved by the A Coruna-Ferrol Research Ethics Committee, under the number 2020/318, on 20 July 2020. In addition, this protocol was registered in Clinical Trials Protocol Registration and Results system on 23 September 2020, available at https:/ / clinicaltrials.gov/ct2/show/NCT04568408. In case any change in the protocol is needed, this will be communicated to the ethics committee with the assigned reference number. These modifications will also be updated in the clinical trials registry.

For each participant, the process of informed consent will be applied. Participants will receive complete verbal and written information about the characteristics of the study and about the implications derived from their participation in it. An information sheet that they can read slowly will be given to each participant, and they will be able to ask any questions they may have. Once it has been ensured that all participants fully understand the information provided, they will decide on whether or not they wish to participate in the study and, if they decide to participate, agree by signing the informed-consent document. The main researcher will maintain the confidentiality of all data collected and the anonymity of each participant. Thus, the Spanish and European Organic Law on the protection of personal data will be respected at all times $[65,66]$. The data of the participants will be collected, encoded, and preserved until the end of the study. Once the study is finished, each participant's data will be stored for future studies if they provide consent. The results and conclusions of this study will be disseminated through their publication in influential scientific journals and their presentation at appropriate conferences.

\section{Discussion}

The validation conducted in this study will allow us to evaluate whether the Xiaomi Mi Smart Band 5 is a device that can be used as a health promoter by allowing sleep self-assessment in the general population. Due to its low price, it is fairly accessible to any user who considers that they may be suffering from a sleep disorder that is potentially worsening their health [32]. Hence, if the data generated by the smart band proves to be reliable, any health professional could evaluate at a glance whether the user is suffering from a sleep disorder, and whether it influences their daily life activities [40,67]. The widespread use of these devices could help users to self-assess their sleep and connect with health professionals in the case of bad sleep quality, which would imply a better prognosis and a longer and higher-quality life expectancy. It is important to highlight some limitations and risks in studies focused on the validation of wearables, since firmware updates may modify the sleep-tracking algorithm, and the intra-wearables' sleep measurements do not perfectly match, even when multiple smart bands worn by the same user are identical.

\section{Conclusions}

This project contributes to the validation of the sleep data obtained through the Xiaomi MiBand 5, making a comparison with the PSG data. Therefore, it is intended to determine whether the sleep parameters (TIB, SOL, WASO, SE, light sleep, deep sleep and REM sleep) are correctly measured through the Xiaomi MiBand 5.

The widespread use of these devices by the current society is not expected to replace the PSG. However, the information obtained by the wristbands about sleep is user-friendly in a domestic context, as this sleep data doesn't need to be processed as in the case of 
the PSG. Thus, the use of these devices can help the population to be able to objectively determine whether they have difficulties in their sleep or not, as well as to promote healthy lifestyle habits, and if necessary, seek the advice of a healthcare professional.

Supplementary Materials: The following are available online athttps:/ /www.mdpi.com/1660-4 601/18/3/1106/s1, File S1: Standard Protocol Items: Recommendations for Interventional Trials (SPIRIT).

Author Contributions: Conceptualization, J.P., P.C.-M., F.J.M.-M. (Francisco Javier Mejuto-Muiño), and B.G.; methodology, J.P., F.J.M.-M. (Francisco José Martínez-Martínez), P.C.-M., and B.G.; investigation, J.P., F.J.M.-M. (Francisco José Martínez-Martínez), P.C.-M., and B.G.; writing-original draft preparation, F.J.M.-M. (Francisco José Martínez-Martínez), P.C.-M., and M.d.C.M.-D.; writing—review and editing, J.P., B.G., L.N.-R., and T.P.; visualization, F.J.M.-M. (Francisco José Martínez-Martínez), P.C.-M., and M.d.C.M.-D.; supervision, J.P., B.G., L.N.-R., T.P., and F.J.M.-M. (Francisco Javier MejutoMuiño); project administration, J.P., B.G., L.N.-R., T.P., and F.J.M.-M. (Francisco Javier Mejuto-Muiño); funding acquisition, P.C.-M. and M.d.C.M.-D. All authors have read and agreed to the published version of the manuscript.

Funding: The authors disclose the receipt of the following financial support for the research, authorship, and/or publication of this article: All the economic costs involved in the study will be borne by the research team. This work is supported in part by grants from the European Social Fund 2014-2020. CITIC (Research Centre of the Galician University System) and the Galician University System (SUG) obtained funds through Regional Development Fund (ERDF), with $80 \%$ from the Operational Program ERDF Galicia 2014-2020 and the remaining 20\% from the Secretaría Xeral de Universidades of the Galician University System (SUG). P.C.M. obtained a scholarship (Ref. ED481A2019/069), and M.D.C.M.-D. obtained a scholarship (Ref. ED481A 2018/205) to develop a Ph.D. thesis. Furthermore, the diffusion and publication of this research are funded by the CITIC as a Research Centre by Galician University System with the support previously mentioned (Ref ED431G 2019/01). In addition, this work is also supported in part by the Ministerio de Ciencia e Innovación $\mathrm{R}+\mathrm{D}+\mathrm{I}$ projects in the framework of national programs of knowledge generation and scientific and technological strengthening of the R+D+I system and challenges of society's oriented R+D+I 2019 call (PID2019-104323RB-C33).

Institutional Review Board Statement: This study was approved by the Research Ethics Committee of A Coruña-Ferrol (ref. 2020/318).

Informed Consent Statement: Informed consent was obtained from all subjects involved in the study. Written informed consent has been obtained from the participants to publish this paper.

Data Availability Statement: Once the data collection process is finished and these have been coded, structured, and analyzed, these data will be provided, provided that the Spanish Data Protection Agency consents, to any researcher who contacts the TALIONIS group's Principal Investigator, Javier Pereira (Javier.pereira@udc.es).

Acknowledgments: We would like to express our gratitude to the participants that have kindly agreed to be part of this study, as well as to the clinical staff working at the sleep unit with which we have worked closely.

Conflicts of Interest: The authors declare that they have no competing interests.

\section{References}

1. Filip, I.; Tidman, M.; Saheba, N.; Bennett, H.; Wick, B.; Rouse, N.; Patriche, D.; Radfar, A. Public health burden of sleep disorders: Underreported problem. J. Public Health 2017, 25, 243-248. [CrossRef]

2. Tester, N.J.; Foss, J.J. Sleep as an Occupational Need. Am. J. Occup. Ther. 2017, 72, 7201347010p1. [CrossRef] [PubMed]

3. Matricciani, L.; Bin, Y.S.; Lallukka, T.; Kronholm, E.; Dumuid, D.; Paquet, C.; Olds, T. Past, present, and future: Trends in sleep duration and implications for public health. Sleep Health 2017, 3, 317-323. [CrossRef] [PubMed]

4. Zhou, G.; Liu, S.; Yu, X.; Zhao, X.; Ma, L.; Shan, P. High prevalence of sleep disorders and behavioral and psychological symptoms of dementia in late-onset Alzheimer disease. Medicine 2019, 98, e18405. [CrossRef]

5. Pavlova, K.M.; Latreille, V. Sleep Disorders. Am. J. Med. 2019, 132, 292-299. [CrossRef]

6. Chen, J.; Waite, L.; Kurina, L.M.; Thisted, R.A.; McClintock, M.; Lauderdale, D.S. Insomnia Symptoms and Actigraph-Estimated Sleep Characteristics in a Nationally Representative Sample of Older Adults. J. Gerontol. Ser. A 2015, 70, 185-192. [CrossRef] 
7. Acquavella, J.; Mehra, R.; Bron, M.; Suomi, J.M.H.; Hess, G.P. Prevalence of narcolepsy and other sleep disorders and frequency of diagnostic tests from 2013-2016 in insured patients actively seeking care. J. Clin. Sleep Med. 2020, 16, 1255-1263. [CrossRef]

8. Hale, L.; Troxel, W.; Buysse, D.J. Sleep Health: An Opportunity for Public Health to Address Health Equity. Annu. Rev. Public Health 2020, 41, 81-99. [CrossRef]

9. Lee, M.; Choh, A.C.; Demerath, E.W.; Knutson, K.L.; Duren, D.L.; Sherwood, R.J.; Sun, S.S.; Chumlea, W.C.; Towne, B.; Siervogel R.M.; et al. Sleep disturbance in relation to health-related quality of life in adults: The fels longitudinal study. J. Nutr. Health Aging 2009, 13, 576-583. [CrossRef]

10. Webb, C.A.; Cui, R.; Titus, C.; Fiske, A.; Nadorff, M.R. Sleep disturbance, Activities of Daily Living, and Depressive Symptoms among Older Sdults. Clin. Gerontol. 2017, 41, 172-180. [CrossRef]

11. Zailinawati, A.-H.; Mazza, D.; Teng, C.L. Prevalence of insomnia and its impact on daily function amongst Malaysian primary care patients. Asia Pac. Fam. Med. 2012, 11, 9. [CrossRef] [PubMed]

12. Puri, S.; Herrick, J.E.; Collins, J.P.; Aldhahi, M.; Baattaiah, B. Physical functioning and risk for sleep disorders in US adults: Results from the National Health and Nutrition Examination Survey 2005-2014. Public Health 2017, 152, 123-128. [CrossRef] [PubMed]

13. Hallit, S.; Hajj, A.; Sacre, H.; Al Karaki, G.; Malaeb, D.; Kheir, N.; Salameh, P.; Hallit, R. Impact of Sleep Disorders and Other Factors on the Quality of Life in General Population. J. Nerv. Ment. Dis. 2019, 207, 333-339. [CrossRef] [PubMed]

14. Abdulah, D.M.; Piro, R.S. Sleep disorders as primary and secondary factors in relation with daily functioning in medical students. Ann. Saudi Med. 2018, 38, 57-64. [CrossRef] [PubMed]

15. Wade, A. The societal costs of insomnia. Neuropsychiatr. Dis. Treat. 2010, 7, 1. [CrossRef] [PubMed]

16. Botteman, M. Health economics of insomnia therapy: Implications for policy. Sleep Med. 2009, 10, S22-S25. [CrossRef]

17. Johnson, D.A.; Billings, M.E.; Hale, L. Environmental Determinants of Insufficient Sleep and Sleep Disorders: Implications for Population Health. Curr. Epidemiol. Rep. 2018, 5, 61-69. [CrossRef]

18. Skaer, T.L.; Sclar, D.A. Economic Implications of Sleep Disorders. Pharmacoeconomics 2010, 28, 1015-1023. [CrossRef]

19. Markwald, R.R.; Bessman, S.C.; Reini, S.A.; Drummond, S.P.A. Performance of a Portable Sleep Monitoring Device in Individuals with High Versus Low Sleep Efficiency. J. Clin. Sleep Med. 2016, 12, 95-103. [CrossRef]

20. de Zambotti, M.; Baker, F.C.; Willoughby, A.R.; Godino, J.G.; Wing, D.; Patrick, K.; Colrain, I.M. Measures of sleep and cardiac functioning during sleep using a multi-sensory commercially-available wristband in adolescents. Physiol. Behav. 2016, 158, 143-149. [CrossRef]

21. Kurina, L.M.; Thisted, R.A.; Chen, J.H.; McClintock, M.K.; Waite, L.J.; Lauderdale, D.S. Actigraphic sleep characteristics among older Americans. Sleep Health 2015, 1, 285-292. [CrossRef] [PubMed]

22. Chamorro, N.; Sellarés, J.; Millán, G.; Cano, E.; Soler, N.; Embid, C.; Montserrat, J.M. An integrated model involving sleep units and primary care for the diagnosis of sleep apnoea. Eur. Respir. J. 2013, 42, 1151-1154. [CrossRef] [PubMed]

23. Merilahti, J.; Korhonen, I. Association between Continuous Wearable Activity Monitoring and Self-Reported Functioning in Assisted Living Facility and Nursing Home Residents. J. Frailty Aging 2016, 5, 225-232. [CrossRef] [PubMed]

24. Shelgikar, A.V.; Anderson, P.F.; Stephens, M.R. Sleep Tracking, Wearable Technology, and Opportunities for Research and Clinical Care. Chest 2016, 446, 732-743. [CrossRef]

25. Griessenberger, H.; Heib, D.P.J.; Kunz, A.B.; Hoedlmoser, K.; Schabus, M. Assessment of a wireless headband for automatic sleep scoring. Sleep Breath. 2013, 17, 747-752. [CrossRef]

26. Smith, M.T.; McCrae, C.S.; Cheung, J.; Martin, J.L.; Harrod, C.G.; Heald, J.L.; Carden, K.A. Use of actigraphy for the evaluation of sleep disorders and circadian rhythm sleep-wake disorders: An American academy of sleep medicine clinical practice guideline. J. Clin. Sleep Med. 2018, 14, 1231-1237. [CrossRef]

27. Withrow, D.; Roth, T.; Koshorek, G.; Roehrs, T. Relation between ambulatory actigraphy and laboratory polysomnography in insomnia practice and research. J. Sleep Res. 2019, 176, e12854. [CrossRef]

28. Faerman, A.; Kaplan, K.A.; Zeitzer, J.M. Subjective sleep quality is poorly associated with actigraphy and heart rate measures in community-dwelling older men. Sleep Med. 2020, 73, 154-161. [CrossRef]

29. Williams, J.M.; Taylor, D.J.; Slavish, D.C.; Gardner, C.E.; Zimmerman, M.R.; Patel, K.; Reichenberger, D.A.; Francetich, J.M.; Dietch, J.R.; Estevez, R. Validity of Actigraphy in Young Adults With Insomnia. Behav. Sleep Med. 2020, 18, 91-106. [CrossRef]

30. Roberts, D.M.; Schade, M.M.; Mathew, G.M.; Gartenberg, D.; Buxton, O.M. Detecting sleep using heart rate and motion data from multisensor consumer-grade wearables, relative to wrist actigraphy and polysomnography. Sleep 2020, 43, 1-15. [CrossRef]

31. Xie, J.; Wen, D.; Liang, L.; Jia, Y.; Gao, L.; Lei, J. Evaluating the validity of current mainstream wearable devices in fitness tracking under various physical activities: Comparative study. J. Med. Internet Res. 2018, 20, e94. [CrossRef] [PubMed]

32. Kubala, A.G.; Barone Gibbs, B.; Buysse, D.J.; Patel, S.R.; Hall, M.H.; Kline, C.E. Field-based Measurement of Sleep: Agreement between Six Commercial Activity Monitors and a Validated Accelerometer. Behav. Sleep Med. 2020, 18, 637-652. [CrossRef] [PubMed]

33. Bravo, P.; Contreras, A.; Perestelo-Pérez, L.; Pérez-Ramos, J.; Málaga, G. Looking for a more participative healthcare: Sharing medical decision making. Rev. Peru. Med. Exp. Salud Publica 2013, 30, 691-697. [PubMed]

34. Cook, J.D.; Prairie, M.L.; Plante, D.T. Utility of the Fitbit Flex to evaluate sleep in major depressive disorder: A comparison against polysomnography and wrist-worn actigraphy. J. Affect. Disord. 2017, 217, 299-305. [CrossRef] [PubMed] 
35. Wan, J.; Gu, X.; Chen, L.; Wang, J. Internet of things for ambientassisted living: Challenges and future opportunities. In Proceedings of the International Conference on Cyber-Enabled Distributed Computing and Knowledge Discovery(CyberC), Nanjing, China, 12-14 October 2017; pp. 354-357.

36. Seo, D.; Yoo, B.; Ko, H. Distributed, Ambient and Pervasive Interactions; Streitz, N., Markopoulos, P., Eds.; Lecture Notes in Computer Science; Springer International Publishing: Cham, Switzerland, 2016; Volume 9749, ISBN 978-3-319-39861-7.

37. Banaee, H.; Ahmed, M.U.; Loutfi, A. Data mining for wearable sensors in health monitoring systems: A review of recent trends and challenges. Sensors 2013, 13, 17472-17500. [CrossRef]

38. Singh, J.; Keer, N. Overview of Telemedicine and Sleep Disorders. Sleep Med. Clin. 2020, 15, 341-346. [CrossRef]

39. Apesteguía, L.; Pina, L.J. Ultrasound-guided core-needle biopsy of breast lesions. Insights Imaging 2011, 2, 493-500. [CrossRef]

40. Mičková, E.; Machová, K.; Dad'ová, K.; Svobodová, I. Does Dog Ownership Affect Physical Activity, Sleep, and Self-Reported Health in Older Adults? Int. J. Environ. Res. Public Health 2019, 16, 3355. [CrossRef]

41. Nieto-Riveiro, L.; Groba, B.; Miranda, M.C.; Concheiro, P.; Pazos, A.; Pousada, T.; Pereira, J. Technologies for participatory medicine and health promotion in the elderly population. Medicine 2018, 97, e10791. [CrossRef]

42. Rundo, J.V.; Downey, R. Polysomnography. In Handbook of Clinical Neurology; Elsevier: Amsterdam, The Netherlands, 2019; Volume 160, pp. 381-392.

43. Jayarathna, T.; Gargiulo, G.D.; Breen, P.P. Continuous vital monitoring during sleep and light activity using carbon-black elastomer sensors. Sensors 2020, 20, 1583. [CrossRef]

44. Noviyanto, A.; Isa, S.M.; Wasito, I.; Arymurthy, A.M. Selecting Features of Single Lead ECG Signal for Automatic Sleep Stages Classification using Correlation-based Feature Subset Selection. Int. J. Comput. Sci. Issues 2011, 8, 139-148.

45. Lewicke, A.; Sazonov, E.; Corwin, M.J.; Neuman, M.; Schuckers, S. Sleep Versus Wake Classification From Heart Rate Variability Using Computational Intelligence: Consideration of Rejection in Classification Models. IEEE Trans. Biomed. Eng. 2008, 55, 108-118. [CrossRef] [PubMed]

46. Fonseca, P.; Long, X.; Radha, M.; Haakma, R.; Aarts, R.M.; Rolink, J. Sleep stage classification with ECG and respiratory effort. Physiol. Meas. 2015, 36, 2027-2040. [CrossRef] [PubMed]

47. Šušmáková, K.; Krakovská, A. Discrimination ability of individual measures used in sleep stages classification. Artif. Intell. Med. 2008, 44, 261-277. [CrossRef] [PubMed]

48. Gharbali, A.A.; Najdi, S.; Fonseca, J.M. Investigating the contribution of distance-based features to automatic sleep stage classification. Comput. Biol. Med. 2018, 96, 8-23. [CrossRef] [PubMed]

49. de Zambotti, M.; Goldstone, A.; Claudatos, S.; Colrain, I.M.; Baker, F.C. A validation study of Fitbit Charge $2^{\mathrm{TM}}$ compared with polysomnography in adults. Chronobiol. Int. 2018, 35, 465-476. [CrossRef] [PubMed]

50. Kahawage, P.; Jumabhoy, R.; Hamill, K.; Zambotti, M.; Drummond, S.P.A. Validity, potential clinical utility, and comparison of consumer and research-grade activity trackers in Insomnia Disorder I: In-lab validation against polysomnography. J. Sleep Res. 2020, 29. [CrossRef]

51. Ameen, M.S.; Cheung, L.M.; Hauser, T.; Hahn, M.A.; Schabus, M. About the Accuracy and Problems of Consumer Devices in the Assessment of Sleep. Sensors 2019, 19, 4160. [CrossRef]

52. El-Amrawy, F.; Nounou, M.I. Are Currently Available Wearable Devices for Activity Tracking and Heart Rate Monitoring Accurate, Precise, and Medically Beneficial? Healthc. Inform. Res. 2015, 21, 315. [CrossRef]

53. Puri, A.; Kim, B.; Nguyen, O.; Stolee, P.; Tung, J.; Lee, J. User Acceptance of Wrist-Worn Activity Trackers Among CommunityDwelling Older Adults: Mixed Method Study. JMIR mHealth uHealth 2017, 5, e173. [CrossRef]

54. Lai, Y.-H.; Huang, F.-F. A Study on the Intention to Use the Wearable Device in Taiwan: A Case Study on Xiaomi Mi Band. In Advances in Intelligent Systems and Computing; Springer: Berlin, Germany, 2018; Volume 661, pp. 283-292. ISBN 9783319676173.

55. Hulley, S.; Cummings, S.; Browner, W.; Grady, D.; Newman, T. Diseño de Investigaciones Clínicas, 4th ed.; Wolters Kluwer Health: Barcelona, Spain, 2014.

56. Chan, A.; Tetzlaff, J.M.; Altman, D.G.; Laupacis, A.; Gøtzsche, P.C.; Krleža-Jerić, K.; Hróbjartsson, A.; Mann, H.; Dickersin, K.; Berlin, J.A.; et al. SPIRIT 2013 Statement: Defining Standard Protocol Items for Clinical Trials. Ann. Intern. Med. 2013, 158, 200. [CrossRef] [PubMed]

57. de Zambotti, M.; Rosas, L.; Colrain, I.M.; Baker, F.C. The Sleep of the Ring: Comparison of the ŌURA Sleep Tracker against Polysomnography. Behav. Sleep Med. 2019, 17, 124-136. [CrossRef] [PubMed]

58. Buysse, D.J.; Reynolds, C.F.; Monk, T.H.; Berman, S.R.; Kupfer, D.J. The Pittsburgh Sleep Quality Index (PSQI): A new instrument for psychiatric research and practice. Psychiatry Res. 1989, 28, 193-213. [CrossRef]

59. Jafari, B.; Mohsenin, V. Polysomnography. Clin. Chest Med. 2010, 31, 287-297. [CrossRef]

60. Iber, C.; Ancoli-Israel, S.; Chesson, A.L.; Quan, S.F. The AASM Manual for the Scoring of Sleep and Associated Events: Rules, Terminology and Technical Specifications; American Academy of Sleep Medicine, Ed.; American Academy of Sleep Medicine: Westchester, NY, USA, 2007.

61. Kemp, B.; Olivan, J. European data format 'plus' (EDF+), an EDF alike standard format for the exchange of physiological data. Clin. Neurophysiol. 2003, 114, 1755-1761. [CrossRef]

62. Cohen, J. Statistical Power Analysis for the Behavioral Sciences, 2nd ed.; Lawrence Erlbaum Associates: Hillsdale, NJ, USA, 1998.

63. Bunce, C. Correlation, Agreement, and Bland-Altman Analysis: Statistical Analysis of Method Comparison Studies. Am. J. Ophthalmol. 2009, 148, 4-6. [CrossRef] 
64. Martin Bland, J.; Altman, D. Statistical methods for assessing agreement between two methods of clinical measurement. Lancet 1986, 327, 307-310. [CrossRef]

65. Agencia Española de Protección de datos Reglamento General de Protección de Datos. Available online: http://www.agpd.es/ portalwebAGPD/temas/reglamento/index-ides-idphp.php (accessed on 26 January 2021).

66. The European Parliament; The Council of the European Union. Regulation (EU) 2016/679 of the European Parliament and of the Council of 27 April 2016 on the Protection of Natural Persons with Regard to the Processing of Personal Data and on the Free Movement of such Data, and Repealing; European Parliament: Brussels, Belgium, 2016; pp. 45-62.

67. Faulkner, S.; Mairs, H. An exploration of the role of the occupational therapist in relation to sleep problems in mental health settings. Br. J. Occup. Ther. 2015, 78, 516-524. [CrossRef] 